$18,12,19$

\title{
Влияние химической модификации поверхности углеродных нанотрубок на их теплопроводность
}

\author{
(C) А.В. Савин ${ }^{1,2}$, О.И. Савина ${ }^{2}$ \\ ${ }^{1}$ Институт химической фризики им. Н.Н. Семенова РАН, \\ Москва, Россия \\ ${ }^{2}$ Российский экономический университет им. Г.В. Плеханова, \\ Москва, Россия \\ E-mail: asavin@center.chph.ras.ru
}

(Поступила в Редакцию 27 июня 2018 г.)

\begin{abstract}
Исследовано влияние на теплопроводность одностенной углеродной нанотрубки частичной химической модификации ее поверхности. Численное моделирование теплопереноса показало, что частичное гидрирование (фторирование) нанотрубки (присоединение атомов водорода, фтора с ее внешней стороны) может приводить к более чем десятикратному уменьшению теплопроводности. С увеличением длины нанотрубки ее теплопроводность растет пропорционально логарифму длины, а коэффициент пропорциональности уменьшается с увеличением плотности присоединенных атомов водорода (фтора). Коэффициент понижения теплопроводности не зависит от длины нанотрубки, но зависит от температуры (чем меньше температура, тем сильнее понижение) и плотности присоединенных атомов $p$. При $p<0.25$ увеличение плотности приводит к монотонному понижению теплопроводности. Максимальное понижение происходит при плотности $p=0.25$. Если гидрировать только одну половину нанотрубки, то эта половина будет иметь меньшую теплопроводность. Такая нанотрубка становится анизотропной и может быть использована как выпрямитель теплопереноса с эффективностью выпрямления, не превышающей двух процентов.

Работа выполнена за счет субсидии, выделенной ИХФ РАН на выполнение государственного задания (тема № 44.7, гос. регистрация АААА-АІ7-117042510268-5). Вычислительные ресурсы предоставлены межведомственным суперкомпьютерным центром РАН.
\end{abstract}

DOI: 10.21883/FTT.2019.02.47145.177

\section{1. Введение}

Углеродные нанотрубки (УНТ) представляют собой цилиндрические макромолекулы диаметром от половины нанометра и длиной до нескольких микрометров. Подобные структуры были получены более 60 лет назад при термическом разложении окиси углерода на железном контакте [1]. Но сами нанотрубки получены только 27 лет назад как побочные продукты синтеза фуллерена $\mathrm{C}_{60}$ [2]. В настоящее время УНТ привлекают внимание благодаря своим уникальным свойствам [3], например, аномально высокой теплопроводностью [4]. Нанотрубка является квазиодномерной молекулярной структурой с ярко выраженными нелинейными свойствами [5].

Целью настоящей работы является исследование влияния на теплопроводность УНТ ее частичного гидрирования, фторирования (валентного присоединения с внешней стороны нанотрубки к части атомов углерода атомов водорода, фтора). Идеальная нанотрубка образована гексагональной решеткой $s p^{2}$ валентных связей $\mathrm{C}-\mathrm{C}$. Присоединение к атому углерода атома водорода (фтора) приводит к локальному изменению типа валентных связей $s p^{2} \rightarrow s p^{3}(\mathrm{C}-\mathrm{C} \rightarrow \mathrm{C}-\mathrm{CH})$. В идеальной решетке валентных связей появляется структурный дефект. Накопление таких дефектов приводит к понижению теплопроводности из-за возникающего до- полнительного рассеяния на них фононов. На примере одностенной УНТ с индексом хиральности $(6,6)$ будет показано, что ее частичное гидрирование, как и фторирование, может приводить к сильному (более чем десятикратному) понижению теплопроводности, величина которого не меняется с увеличением длины нанотрубки, а зависит от температуры и концентрации присоединенных атомов.

Также будет рассмотрена возможность использования частично гидрированной нанотрубки в качестве выпрямителя теплопереноса. Если гидрировать только одну половину нанотрубки, то эта половина будет иметь меньшую теплопроводность. Такая нанотрубка становится анизотропной и может быть использована как выпрямитель теплопереноса с эффективностью выпрямления, не превышающей двух процентов. Будет показано, что в такой нанотрубке поток тепла в одну сторону может превосходить поток тепла в другую только на два процента.

\section{2. Модель углеродной нанотрубки}

Рассмотрим УНТ с индексом хиральности $(6,6)$, представленную на рис. 1. Цилиндрическая структура нанотрубки образуется периодическим повторением вдоль оси $x$ поперечных циклических зигзагообразных цепочек 


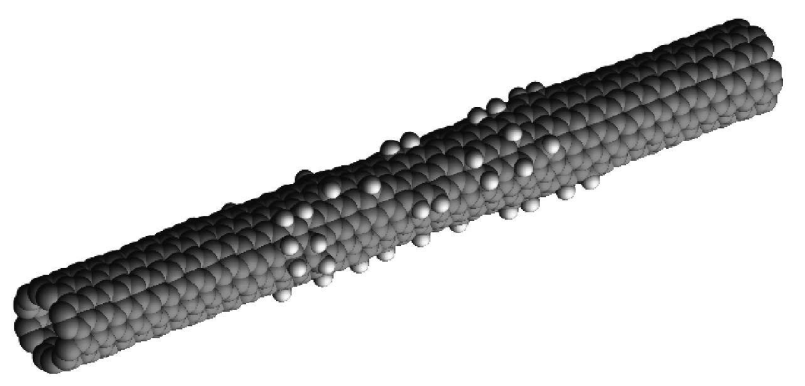

Рис. 1. Одностенная углеродная нанотрубка с индексом хиральности $(6,6)$ (нанотрубка типа „кресло“ с диаметром $D=0.805 \mathrm{~nm}$ ) с гидрированной центральной частью (плотность присоединения атомов водорода $p=0.1$ ). Атомы водорода показаны светлыми, углерода - серыми шариками.

из 24 атомов углерода (поперечный диаметр нанотрубки $D=8.05 \AA$, продольный шаг $a=2.46 \AA$ ).Пусть индекс $n$ нумерует поперечные кольца атомов со структурой „кресло“, тогда положения атомов каждого кольца (поперечной ячейки нанотрубки) можно описать вектором размерности 72: $\mathbf{u}_{n}=\left\{\left(x_{n, k}, y_{n, k}, z_{n, k}\right)\right\}_{k=1}^{24}$ (индекс $n$ нумерует атомы углерода вдоль, индекс $k-$ поперек нанотрубки).

Гамильтониан нанотрубки имеет вид

$$
H=\sum_{n=-\infty}^{+\infty} \sum_{k=1}^{24}\left[\frac{1}{2} M_{(n, k)}\left(\dot{\mathbf{v}}_{(n, k)}, \dot{\mathbf{v}}_{(n, k)}\right)+P_{(n, k)}\right] \text {, }
$$

где $M_{\alpha}-$ масса атома с индексом $\alpha=(n, k)$, $\mathbf{v}_{\alpha}=\left(x_{n, k}(t), y_{n, k}(t), z_{n, k}(t)\right)-$ вектор, задающий положения атома. Слагаемое $P_{\alpha}$ описывает взаимодействие атома с индексом $\alpha$ с его соседними атомами. Этот потенциал зависит от изменения длин валентных связей, углов и изменения двугранных углов между плоскостями, формируемыми тремя соседними атомами.

Динамика идеальной нанотрубки хорошо описывается при помощи потенциалов взаимодействия [6,7]: деформация валентной связи $\mathrm{C}-\mathrm{C}-$ потенциалом

$$
V(r)=\epsilon_{0}\left[e^{-\alpha\left(r-r_{0}\right)}-1\right]^{2}
$$

где $r$ - текущая длина связи, $r_{0}$ - ее равновесное значение, $\epsilon_{0}-$ энергия связи, параметр $\alpha$ задает жесткость связи $K=2 \epsilon_{0} \alpha^{2}$; деформация валентного угла $\mathrm{C}-\mathrm{C}-\mathrm{C}-$ потенциалом

$$
U\left(\mathbf{u}_{1}, \mathbf{u}_{2}, \mathbf{u}_{3}\right)=U(\varphi)=\epsilon_{1}\left(\cos \varphi-\cos \varphi_{0}\right)^{2},
$$

где косинус валентного угла определяется выражением $\cos \varphi=-\left(\mathbf{v}_{1}, \mathbf{v}_{2}\right) /\left|\mathbf{v}_{1}\right|\left|\mathbf{v}_{2}\right|$, векторы $\mathbf{v}_{1}=\mathbf{u}_{2}-\mathbf{u}_{1}$, $\mathbf{v}_{2}=\mathbf{u}_{3}-\mathbf{u}_{2}$ (вектора $\mathbf{u}_{1}, \mathbf{u}_{2}, \mathbf{u}_{3}$ задают координаты атомов углерода, образующих валентный угол), $\varphi_{0}-$ равновесное значение угла, энергия $\epsilon_{1}$ характеризует
Таблица 1. Значения параметров потенциала (2) для различных типов валентной связи

\begin{tabular}{c|c|c|c|c|c}
\hline $\begin{array}{c}\text { Валентная } \\
\text { связь }\end{array}$ & $\mathrm{C}-\mathrm{C}$ & $\mathrm{C}-\mathrm{CH}$ & $\mathrm{C}-\mathrm{H}$ & $\mathrm{C}-\mathrm{CF}$ & $\mathrm{C}-\mathrm{F}$ \\
\hline$\epsilon_{0}(\mathrm{eV})$ & 4.9632 & 4.01 & 4.28 & 4.01 & 5.38 \\
$r_{0}(\AA)$ & 1.418 & 1.51 & 1.11 & 1.53 & 1.36 \\
$\alpha\left(\AA^{-1}\right)$ & 1.7889 & 1.8 & 1.8 & 1.8 & 2.0 \\
$K(\mathrm{~N} / \mathrm{m})$ & 508.9 & 416.3 & 444.3 & 416.3 & 689.5
\end{tabular}

Таблица 2. Значения параметров потенциала валентного угла (3) для различных валентных связей

\begin{tabular}{c|c|c|c|c|c}
\hline $\begin{array}{c}\text { Валентный } \\
\text { угол }\end{array}$ & $\mathrm{C}-\mathrm{C}-\mathrm{C}$ & $\mathrm{C}-\mathrm{CH}-\mathrm{C}$ & $\mathrm{C}-\mathrm{CF}-\mathrm{C}$ & $\mathrm{C}-\mathrm{C}-\mathrm{H}$ & $\mathrm{C}-\mathrm{C}-\mathrm{F}$ \\
\hline$\epsilon_{0}(\mathrm{eV})$ & 1.3143 & 1.3 & 1.3 & 1.0 & 1.0 \\
$\varphi_{0}$ & $120^{\circ}$ & $111^{\circ}$ & $112.7^{\circ}$ & $109.5^{\circ}$ & $109.2^{\circ}$
\end{tabular}

жесткость угла. Деформация двугранных и торсионных углов описывается потенциалом

$$
W\left(\mathbf{u}_{1}, \mathbf{u}_{2}, \mathbf{u}_{3}, \mathbf{u}_{4}\right)=\epsilon_{2}(1+\cos \phi),
$$

где $\cos \phi=\left(\mathbf{v}_{1}, \mathbf{v}_{2}\right) /\left|\mathbf{v}_{1}\right|\left|\mathbf{v}_{2}\right|$, векторы $\mathbf{v}_{1}=\left(\mathbf{u}_{2}-\mathbf{u}_{1}\right)\left(\mathbf{u}_{3}-\mathbf{u}_{2}\right)$, $\mathbf{v}_{2}=\left(\mathbf{u}_{3}-\mathbf{u}_{2}\right)\left(\mathbf{u}_{4}-\mathbf{u}_{3}\right)$, энергия $\epsilon_{2}=0.499 \mathrm{eV}$ характеризует жесткость угла (векторы $\mathbf{u}_{1}, \ldots, \mathbf{u}_{4}$ задают положения атомов, образующих торсионный или двугранный угол).

Присоединение атома водорода (фтора) к атому углерода меняет тип всех валентных связей этого атома. Поэтому здесь необходимо модифицировать потенциалы взаимодействия. Значения параметров потенциалов взаимодействия (2) и (3) для различных связей даны в табл. 1 и 2. Деформации торсионных и двугранных углов, в образовании грани которых не участвуют атомы углерода с присоединенными атомами водорода (фтора), опишем потенциалом (4). Если к одному из атомов углерода, образующих грань (ребро) торсионного (двугранного) угла, присоединен атом водорода (фтора), будем использовать потенциал

$$
W\left(\mathbf{u}_{1}, \mathbf{u}_{2}, \mathbf{u}_{3}, \mathbf{u}_{4}\right)=\epsilon_{3}(1+\cos 3 \phi)
$$

с параметром $\epsilon_{3}=0.03 \mathrm{eV}$. Обоснование выбранных значений параметров потенциалов взаимодействия дано в работах [7-9].

\section{3. Моделирование теплопроводности}

Для прямого моделирования переноса тепла рассмотрим конечную нанотрубку из $N$ поперечных ячеек (длина нанотрубки $L=(N-1) a)$. Зафиксируем положения атомов из ее краевых ячеек с номерами $n=1, n=N$. Поместим ее $N_{+}$первых ячеек в термостат Ланжевена с температурой $T_{+}$, а $N_{-}$последних - в термостат 
с температурой $T_{-}<T_{+}$. Тогда динамика нанотрубки будет описываться системой уравнений движения

$$
\begin{gathered}
\mathbf{M}_{n} \ddot{\mathbf{u}}_{n}=-\frac{\partial}{\partial \mathbf{u}_{n}} H-\Gamma \mathbf{M}_{n} \dot{\mathbf{u}}_{n}+\Xi_{n}^{+}, \quad n=2, \ldots, N_{+}, \\
\mathbf{M}_{n} \ddot{\mathbf{u}}_{n}=-\frac{\partial}{\partial \mathbf{u}_{n}} H, \quad n=N_{+}+1, \ldots, N-N_{-}, \\
\mathbf{M}_{n} \ddot{\mathbf{u}}_{n}=-\frac{\partial}{\partial \mathbf{u}_{n}} H-\Gamma \mathbf{M}_{n} \dot{\mathbf{u}}_{n}+\Xi_{n}^{-}, \\
n=N-N_{-}+1, \ldots, N-1,
\end{gathered}
$$

где $\mathbf{M}_{n}$ - диагональная матрица масс атомов $n$-ой ячейки, $\mathbf{u}_{n}-72$-мерный вектор, задающий координаты ее атомов, коэффициент трения $\Gamma=1 / t_{r}$ (время релаксации $\left.t_{r}=0.4 \mathrm{ps}\right), \Xi_{n}^{ \pm}=\left\{\xi_{n, k}\right\}_{k=1}^{72}-72$-мерный вектор нормально распределенных случайных сил с условием нормировки

$$
\left\langle\xi_{n, i}^{ \pm}\left(t_{1}\right) \xi_{l, j}^{ \pm}\left(t_{2}\right)\right\rangle=2 M_{n, i} k_{\mathrm{B}} T_{ \pm} \Gamma \delta_{n l} \delta_{i j} \delta\left(t_{1}-t_{2}\right)
$$

$\left(k_{\mathrm{B}}-\right.$ постоянная Больцмана).

Возьмем начальные условия системы (6) соответствующими основному состоянию нанотрубки и численно проинтегрируем систему уравнений движения до момента времени, когда в наноленте образуется стационарное распределение температуры. Затем найдем распределение температуры вдоль нанотрубки

$$
\begin{gathered}
T_{n}=\lim _{t \rightarrow \infty} \frac{1}{72 k_{\mathrm{B}} t} \int_{0}^{t}\left(\mathbf{M}_{n} \dot{\mathbf{u}}_{n}(\tau), \dot{\mathbf{u}}_{n}(\tau)\right) d \tau, \\
n=2, \ldots, N-1 .
\end{gathered}
$$

Типичное распределение температуры вдоль нанотрубки представлено на рис. 2.

Стационарный температурный профиль (7) позволяет найти среднее значение потока энергии $J$. Действительно, если бы нанотрубка целиком была бы помещена в термостат Ланжевена с температурой $T$, то каждый ее сегмент имел бы температуру $T_{n}=T$ вследствие баланса между приходом энергии от действия случайных сил и ее потерей на трение. Поэтому средний приток энергии из термостата в $n$-ячейку может быть определен как

$$
\Gamma\left\langle\left(\mathbf{M}_{n} \dot{\mathbf{u}}_{n}, \dot{\mathbf{u}}_{n}\right)\right\rangle=72 k_{b} T_{n} / t_{r}
$$

(приток энергии в единицу времени равен работе сил трения). Если в термостаты помещены только концы нанотрубки, то возникает дополнительный поток энергии в ее центральную часть. Энергия от более теплого левого края будет идти в более холодный правый край. В результате этого температура на левом крае нанотрубки уменьшится $\left(T_{n}<T_{+}\right)$, а на правом возрастет $\left(T_{n}>T_{-}\right)-$см. рис. 2 . Поэтому, изменение распределения температуры на взаимодействующих с

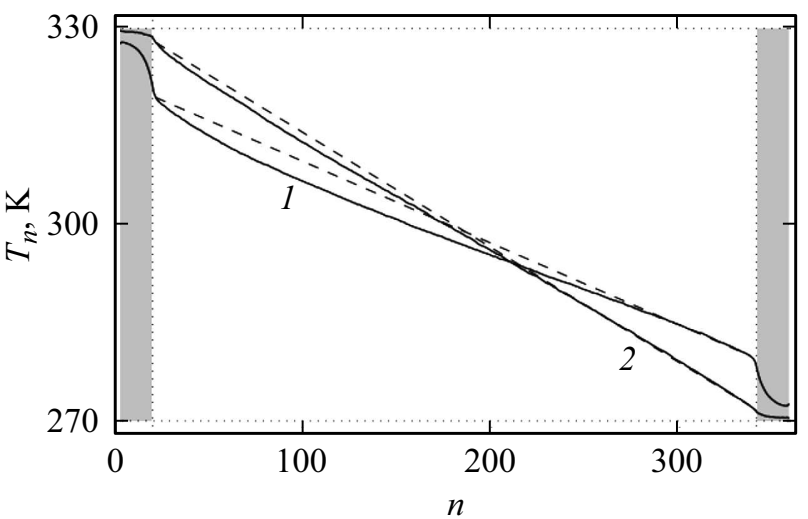

Рис. 2. Распределение вдоль нанотрубки температуры $T_{n}$, возникающее при помещении ее концов в термостаты Ланжевена температуры $T_{ \pm}=300 \pm 30 \mathrm{~K}$. Число поперечных ячеек $N=360$ (длина нанотрубки $L=(N-1) a=88.3 \mathrm{~nm}$ ), число концевых ячеек, взаимодействующих с термостатами, $N_{ \pm}=20$ (области, взаимодействующие с термостатами, выделены серым цветом). Кривая 1 дает распределение температуры для идеальной нанотрубки, кривая 2 - для нанотрубки с гидрированной центральной частью (плотность присоединенных атомов водорода $p=0.1$ ), пунктирные прямые показывают внутренние линейные градиенты температуры, используемые для нахождения коэффициента теплопроводности $\kappa\left(N_{i}\right)$.

термостатами краях нанотрубки позволяет найти поток энергии через ее центральную часть

$$
J t_{r} / 72 a k_{\mathrm{B}}=\sum_{n=2}^{N_{+}}\left(T_{+}-T_{n}\right)=\sum_{N-N_{-}+1}^{N-1}\left(T_{n}-T_{-}\right) .
$$

Если число ячеек взаимодействующих с термостатами концов нанотрубки совпадают $\left(N_{+}=N_{-}=N_{ \pm}\right)$, то формула для теплового потока принимает вид

$$
J=\frac{36 a k_{B}}{t_{r}} \sum_{n=2}^{N_{ \pm}}\left(T_{+}-T_{-}-T_{n}+T_{N+1-n}\right) .
$$

Образующийся в центральной части нанотрубки градиент температуры может быть использован для нахождения коэффициента теплопроводности

$$
\kappa\left(N_{i}\right)=\left(N_{i}-1\right) J /\left(T_{N_{+}+1}-T_{N-N_{-}}\right) S,
$$

где $N_{i}=N-N_{-}-N_{+}$- число поперечных ячеек в центральной части нанотрубки, $S=\pi\left(R+r_{c}\right)^{2}-$ площадь поперечного сечения нанотрубки $(R=D / 2=4.025 \AA$ радиус нанотрубки, $r_{c}=1.85 \AA$ - радиус Ван-дерВаальса атома углерода).

\section{4. Понижение теплопроводности при гидрировании (фторировании) нанотрубки}

Для оценки влияния химической модификации поверхности нанотрубки на ее теплопроводность рас- 
смотрим конечную нанотрубку из $N$ поперечных ячеек. Зафиксируем положения атомов из ее конечных ячеек $n=1$ и $n=N$. Концы нанотрубки из $N_{ \pm}=20$ ячеек поместим в термостаты Ланжевена температуры $T_{ \pm}=(1 \pm 0.1) T$, где $T-$ температура, для которой нужно получить значение теплопроводности. Для моделирования гидрирования (фторирования) к каждому атому углерода из внутренних поперечных ячеек нанотрубки $N_{+}<n \leq N-N_{-}$случайно, с вероятностью $p$, присоединим с внешней стороны атом водорода (фтора) - см. рис. 1. Параметр $p \in[0,1]$ задает плотность присоединения атомов водорода (фтора). Затем проведем численное моделирование теплопереноса вдоль нанотрубки, найдем распределение температуры $\left\{T_{n}\right\}_{n=2}^{N-1}$ (см. рис. 2), величину теплового потока $J$ и коэффициент теплопроводности $\kappa\left(N_{i}\right)$. При вычислениях все результаты усреднялись по 256 случайным реализациям гидрирования (фторирования) центральной части нанотрубки.

Численное моделирование показало, что коэффициент теплопроводности идеальной нанотрубки (плотность присоединенных атомов $p=0$ ) монотонно увеличивается с увеличением ее длины: $\kappa(N) \sim \ln N$ при $N \rightarrow \infty-$ см. рис. 3. При длинах $L<640 \mathrm{~nm}$ (при $N \leq 2600$ ) теплопроводность растет явно медленнее любой степенной функции длины, лучше всего рост аппроксимируется логарифмом длины. Отметим, что в более ранней работе [10] для нанотрубок длины $L<100 \mathrm{~nm}$ был получен степенной рост теплопроводности, $\kappa(N) \sim N^{\beta}$ при $N \rightarrow \infty$, с показателем $\beta \in(0,0.4]$. Полученная степенная асимптотика связана с некорректным использованием при моделировании теплопереноса детерминированного термостата Нозе-Хувера. Более аккуратное моделирование с использованием стохастического термостата Ланжевена и более длинных нанотрубок дает логарифмический рост теплопроводности.

Гидрирование (фторирование) нанотрубки приводит к существенному понижению ее теплопроводности. Моделирование теплопереноса вдоль гидрированной с постоянной плотностью $p$ нанотрубки показало, что здесь теплопроводность также монотонно растет пропорционально логарифму ее длины: $\kappa(N, p) \sim A(p) \ln N$, при $N \rightarrow \infty$, но показатель пропорциональности $A(p)$ уменьшается при увеличении плотности гидрирования (фторирования) $p$. Так при температуре $T=300 \mathrm{~K}$ коэффициент пропорциональности $A=93 \mathrm{~W} / \mathrm{mK}$ при $p=0$ и $A=8 \mathrm{~W} / \mathrm{mK}$ при $p=0.1$ (см. рис. 3 ).

Определим коэффициент понижения теплопроводности как отношение теплопроводности гидрированной нанотрубки к теплопроводности идеальной нанотрубки той же длины: $c(N, p)=\kappa(N, p) / \kappa(N, 0)$. Как видно из рис. $3, b$, коэффициент понижения стремится к ненулевому значению при увеличении длины нанотрубки: $c(N, p) \rightarrow c(p)>0$ при $N \rightarrow \infty$. При плотности гидрирования $p=0.1$, температуре $T=300 \mathrm{~K}$ коэффициент понижения теплопроводности $c \approx 0.1$.
Увеличение плотности гидрирования (фторирования) приводит к монотонному уменьшению теплопроводности нанотрубки (см. рис. 4). Коэффициент понижения

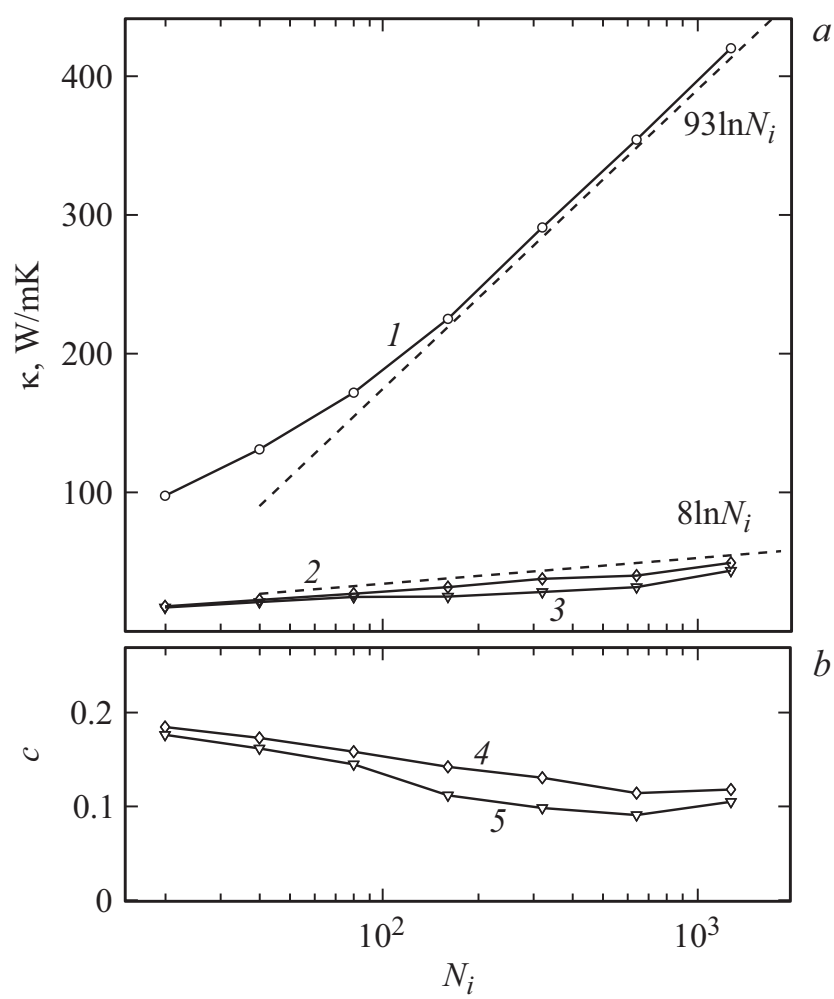

Рис. 3. Зависимость (a) коэффициента теплопроводности $\kappa$ одностенной УНТ с индексом хиральности $(6,6)$ от ее безразмерной длины $N_{i}$ (числа поперечных ячеек) при температуре $T=300 \mathrm{~K}$ для идеальной нанотрубки (кривая 1), для нанотрубки гидрированной (кривая 2), фторированной (кривая 3) с плотностью присоединенных атомов $p=0.1$. Штриховые наклонные линии дают асимптотики $\kappa=93 \ln N_{i}$ и $\kappa=8 \ln N_{i} \mathrm{~W} / \mathrm{mK}$. Часть (b) дает зависимость коэффициента понижения теплопроводности $c$ от $N_{i}$ при гидрировании и фторировании нанотрубки (кривые 4 и 5 ).

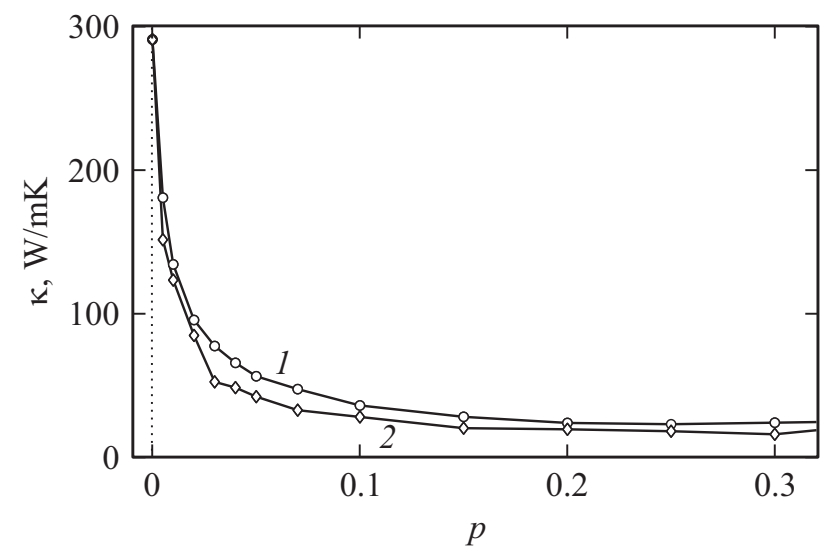

Рис. 4. Зависимость коэффициента теплопроводности $\kappa$ гидрированной (кривая 1) и фторированной (кривая 2) одностенной УНТ с индексом хиральности $(6,6)$ от плотности присоединенных атомов $p$. Число поперечных ячеек нанотрубки $N_{i}=320$, температура $T=300 \mathrm{~K}$. 


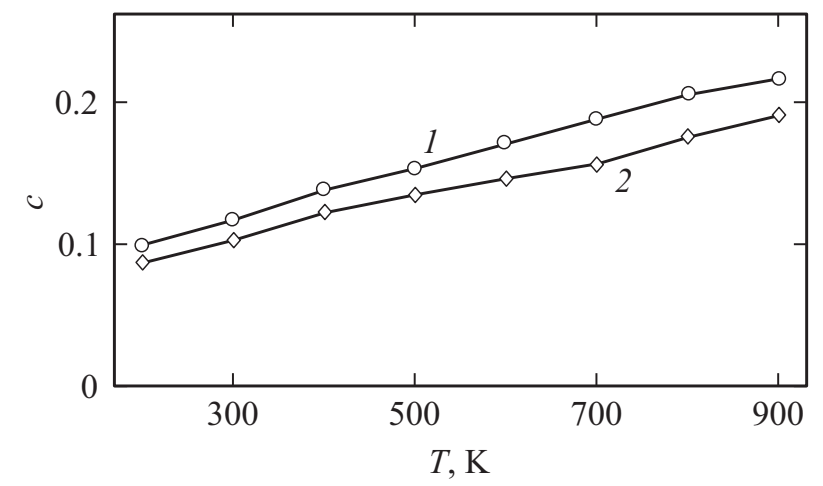

Рис. 5. Зависимость коэффициента понижения теплопроводности $c$ от температуры $T$ дли гидрированной (кривая 1 ) и фторированной (кривая 2) одностенной УНТ с индексом хиральности $(6,6)$. Плотность присоединенных атомов $p=0.1$.

теплопроводности слабо зависит от длины нанотрубки, но существенно зависит от плотности присоединения атомов водорода (фтора). Максимальное понижение происходит при плотности $p=0.25$ (коэффициент понижения $c=0.08$ при гидрировании и $c=0.06$ при фторировании нанотрубки). Дальнейшее увеличение плотности присоединения атомов водорода (фтора) приводит уже к росту теплопроводности, так как структура нанотрубки начинает приближаться к регулярной структуре нанотрубки графона $\mathrm{C}_{2} \mathrm{H}$ (монослоя графена, насыщенного водородом с одной стороны [11-13]).

Коэффициент понижения теплопроводности монотонно зависит от температуры: чем меньше температура, тем сильнее происходит понижение (см. рис. 5). Это связано с фононным механизмом подавления теплопроводности - рассеяния фононов на структурных дефектах решетки. С ростом температуры нелинейность динамики нанотрубки возрастает, что приводит к уменьшению вклада фононов в теплоперенос и, следовательно, к уменьшению потери энергии от рассеяния фононов.

Фторирование нанотрубки приводит к более сильному понижению теплопроводности, чем ее гидрирование. Это связано с более высокой массой фтора к структурному дефекту валентных связей добавляется локальное увеличение масс присоединенных атомов, что приводит к увеличению рассеяния фононов.

\section{5. Частично гидрированная нанотрубка как тепловой выпрямитель}

Если гидрировать только одну половину нанотрубки, ее поперечные ячейки с номерами $N_{+}<n \leq N / 2$, то получим нанотрубку, левая половина которой имеет значительно меньшую теплопроводность, чем правая. Нанотрубка становится анизотропной, поэтому здесь можно ожидать эффекта выпрямления теплопереноса зависимости величины теплового потока от его направления (от знака градиента температуры). Детальный обзор возможных выпрямителей теплопереноса дан в обзоре [14]. В работах [15-17] в качестве выпрямителей теплопереноса были рассмотрены наполовину гидрированные углеродные наноленты и нанотрубки.

Для моделирования выпрямления теплопереноса рассмотрим нанотрубку с фиксированными краями из $N$-поперечных ячеек. Концы нанотрубки из $N_{ \pm}=20$ ячеек поместим в термостаты Ланжевена температуры $T_{ \pm}=300 \pm 100 \mathrm{~K}$ (для моделирования теплопереноса слева направо) и температуры $T_{ \pm}=300 \mp 100 \mathrm{~K}$ (для моделирования теплопереноса в обратном направлении). Левую половину нанотрубки, т.е. ячейки с номерами $N_{+}<n \leq N / 2$, гидрируем с плотностью присоединенных атомов водорода $p=0.1$. Результаты моделирования будем усреднять по 256 независимым случайным реализациям присоединения атомов водорода.

Рассмотрим, как зависит величина теплового потока от направления теплопереноса. Возникающие вдоль нанотрубки распределения температуры показаны на рис. 6. Пусть $J_{+}>0-$ величина теплового потока при теплопереносе слева направо, а $J_{-}<0-$ при теплопереносе справа налево. Определим коэффициент анизотропии (выпрямления) теплопереноса

$$
\epsilon_{a}=\left(J_{+}+J_{-}\right) /\left(J_{+}-J_{-}\right) .
$$

Параметр анизотропии $\epsilon_{a} \in[-1,1]:$ при $\epsilon_{a}=0$ анизотропия отсутствует $\left(J_{+}=-J_{-}\right)$, при $\epsilon_{a}>0$ тепловой поток выше при теплопереносе слева направо $\left(J_{+}>-J_{-}\right)$и наоборот при $\epsilon_{a}<0$. При $\epsilon_{a}= \pm 1$ имеет место полное $100 \%$ выпрямление - теплоперенос возможен только в одном направлении.

Численное моделирование показало, что величина теплового потока слева направо всегда немного выше величины потока в обратном направлении $\left(J_{+}>-J_{-}\right)$.

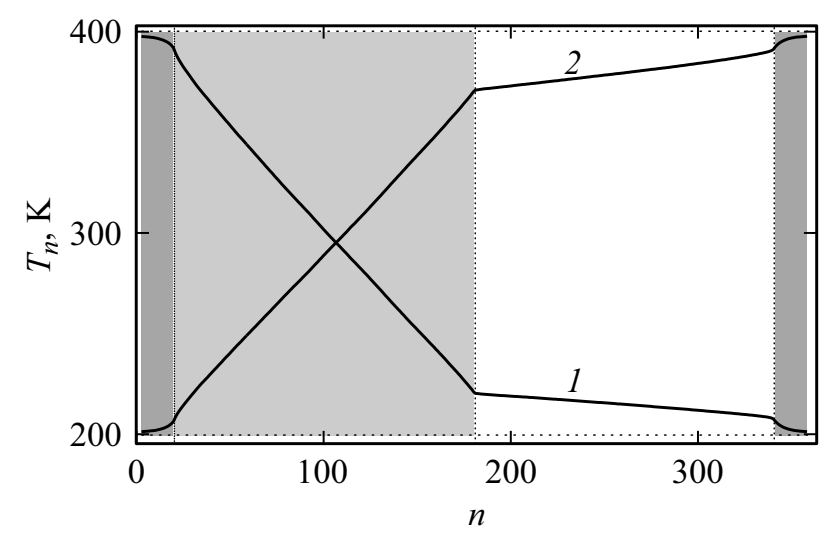

Рис. 6. Распределение вдоль наполовину гидрированной нанотрубки температуры $T_{n}$, возникающее при помещении ее концов в термостаты Ланжевена температуры $T_{ \pm}=300 \pm 100 \mathrm{~K}$ (кривая 1) и $T_{ \pm}=300 \mp 100 \mathrm{~K}$ (кривая 2). Число поперечных ячеек $N=360$ (длина нанотрубки $L=(N-1) a=88.3 \mathrm{~nm}$ ), число концевых ячеек, взаимодействующих с термостатами, $N_{ \pm}=20$. Области, взаимодействующие с термостатами, выделены темно-серым цветом, гидрированная левая половина нанотрубки показана светло-серым цветом (плотность присоединенных атомов водорода $p=0.1)$. 
При $N=80$ коэффициент анизотропии теплопереноса $\epsilon_{a}=0.007$, при $N=120$ коэффициент $\epsilon=0.009$, при $N=200$ уже $\epsilon=0.016$, а при $N=360$ анизотропия $\epsilon=0.019$. Если при $N=360$ гидрировать только ячейки с номерами $N_{+}<n \leq N / 4$, то анизотропия $\epsilon=0.013$, а при гидрировании ячеек с $N_{+}<n \leq 3 N / 4$ анизотропия $\epsilon=0.011$.

Таким образом, прямое моделирование теплопереноса в частично гидрированной нанотрубке показывает возможность, максимум, 2\% выпрямления теплового потока. Отметим, что в работах [15-17] при моделировании теплопереноса было получено 20-30\% выпрямление теплового потока! Это связано с тем, что при моделировании в этих работах был использован детерминированный термостат Нозе-Хувера, который при моделировании неравновесной динамики может приводить к многочисленным артефактам [18-20].

\section{6. Заключение}

Проведенное численное моделирование показывает, что гидрирование (фторирование) углеродных нанотрубок может приводить к более чем десятикратному уменьшению их теплопроводности. С увеличением длины нанотрубки ее теплопроводность растет пропорционально логарифму длины, а коэффициент пропорциональности уменьшается с увеличением плотности присоединенных атомов водорода (фтора). Коэффициент понижения теплопроводности не зависит от длины нанотрубки, но зависит от температуры (чем меньше температура, тем сильнее понижение) и плотности присоединенных атомов $p$. При $p<0.25$ увеличение плотности приводит к монотонному понижению теплопроводности. Максимальное понижение происходит при плотности $p=0.25$. Если гидрировать только одну половину нанотрубки, то эта половина будет иметь меньшую теплопроводность. Такая нанотрубка становится анизотропной и может быть использована как выпрямитель теплопереноса с эффективностью выпрямления, не превышающей двух процентов.

\section{Список литературы}

[1] Л. В. Радушкевич, В. М. Лукьянович. Журн. физ. хим. 26, 1, 88 (1952)

[2] S. Iijima. Nature 354, 56 (1991).

[3] А.В. Елецкий. УФН 172, 4, 401 (2002).

[4] P. Kim, L. Shi, A. Majumdar, P.L. McEuen. Phys. Rev. Lett. 87, 215502 (2001).

[5] А.В. Савин, О.И. Савина. ФТТ 46, 2, 372 (2004).

[6] A.V. Savin, B. Hu, Y.S. Kivshar. Phys. Rev. B 80, 195423 (2009).

[7] A.V. Savin, Y.S. Kivshar, B. Hu. Phys. Rev. B 82, 195422 (2010).

[8] W.D. Cornell, P. Cieplak, C.I. Bayly, I.R. Gould, K.M. Merz, D.M. Ferguson, D.C. Spellmeyer, T. Fox, J.W. Caldwell, P.A. Kollman. J. Am. Chem. Soc. 117, 5179 (1995).
[9] G.M. Chechin, S.V. Dmitriev, I.P. Lobzenko, D.S. Ryabov. Phys. Rev. B 90, 045432 (2014).

[10] G. Zhang, B. Li. J. Chem. Phys. 123, 114714 (2005).

[11] J. Zhou, Q. Wang, Q. Sun, X.C. Chen, Y. Kawazoe, P. Jena. Nano Lett. 9, 11, 3867 (2009).

[12] R. Balog, B. Jorgensen, L. Nilsson, M. Andersen, E. Rienks, M. Bianchi, M. Fanetti, E. Lægsgaard, A. Baraldi, S. Lizzit, Z. Sljivancanin, F. Besenbacher, B. Hammer, T.G. Pedersen, P. Hofmann, L. Hornekær. Nature Mater. 9, 315 (2010).

[13] D.W. Boukhvalov. Physica E 43, 1, 199 (2010).

[14] N.A. Roberts, D.G. Walker. International J. Therm. Sci. 50, 648 (2011).

[15] A. Rajabpour, S.M. Vaez Allaei, F. Kowsary. Appl. Phys. Lett. 99, 051917 (2011).

[16] K. Gordiz, S.M.V. Allaei. J. Appl. Phys. 115, 163512 (2014).

[17] M. Shavikloo, S. Kimiagar. Comput. Mater. Sci. 139, 330 (2017).

[18] A. Fillipov, B. Hu, B. Li, A. Zeltser. J. Phys. A 31, 7719 (1998).

[19] F. Legoll, M. Luskin, R. Moeckel. Nonlinearity 22, 1673 (2009).

[20] J. Chen, G. Zhang, B.W. Li. J. Phys. Soc. Jpn 79, 074604 (2010).

Редактор Ю.Э. Китаев 